\title{
ON THE GAUSS MAP OF MINIMAL SURFACES IMMERSED IN $R^{n}$
}

\author{
By Masahiko Fujiki
}

\section{Introduction.}

The Gauss map of a minimal surface $M$ in $R^{n}$ can be considered as a holomorphic mapping from $M$ to the complex quadric $Q_{n-2}$ in the complex projective space $C P^{n-1}$ with the Fubini-Study metric of constant curvature 2. This paper is devoted to the question, "If a minimal surface $M$ in $R^{n}$ has a constant curvature $\hat{K}$ in its Gaussian image, what values of $\hat{K}$ can be possible?".

This question comes from Ricci's classical theorem;

There exists a minimal surface in $R^{3}$ which is isometric with $M$ iff $\left(M, d s^{2}\right)$ satisfies Ricci condition:

(i) Gaussian curvature $K$ of $M$ is negative,

(ii) the new metric $d \tilde{s}^{2}=\sqrt{-K} d s^{2}$ is flat on $M$.

The condition (ii) is known to be equivalent to the condition " $\hat{K} \equiv 1$ ". (see Lawson [2])

Concerning the question, the following are well-known;

(a) If $\hat{K} \equiv 1$, then $M$ must lie fully in $R^{3}$ or $R^{6}$. And all the minimal surfaces isometric to $M$ make a two parameter family. (Lawson [2])

(b) Minimal surfaces in $R^{4}$ which have constant curvature $\hat{K}$ in their Gaussian images are classified as follows;

i. $\hat{K} \equiv 1$, and $M$ lies in some affine $R^{3}$,

ii. $\hat{K} \equiv 2$, and $M$ is a holomorphic curve in $C^{2}$.

Here $C^{2}$ means $R^{4}$ with some orthogonal complex structure. (Osserman-Hoffman [5])

(c) And in $R^{5}$,

i. $\hat{K} \equiv 1$ or 2 , and $M$ lies in $R^{4}$ (these are the cases (b).)

ii. $\hat{K} \equiv 1 / 2$, and the Gaussian image of $M$ can be represented locally as;

$$
1 / 2\left(1-w^{4}, i+i w^{4}, 2 w+2 w^{3}, 2 i w-2 i w^{3}, 2 \sqrt{3} i w^{2}\right)
$$

(Masal'tsev [4])

To get these results, Calabi's theorem [1] plays the main role. Using the

Received March 11, 1985 
method in [2], [4] and [5], following results are obtained;

THEOREM A. For every positive integer $m$, there exists a minmal surface with $\hat{K} \equiv 1 / m$ in $2 m+1$ dimensional Euclidean space.

THEOREM B. For every integer $m \geqq 5$, there exists a mmmal surface with $\hat{K} \equiv 2 /(2 m-1)$ in $2 m$ dimensional Euclidean space.

THeOREM C. Let $k=3,5$, or 7 . Then there exists a minmal surface $M$ with $\hat{K} \equiv 2 / k$ in $k+3$ dimensional Euclidean space.

The author wishes to express his hearty thanks to Professor S. Tanno and the referee for their valuable suggestions.

\section{Preliminaries.}

Let $M$ be a surface immersed in $R^{n}$. It means that there exists a conformal immersion

$$
X: S \longrightarrow R^{n}, \quad X=\left(X_{1}, X_{2}, \cdots, X_{n}\right)
$$

where $S$ is a Riemann surface. Here we define the Gauss map $g$ as follows;

$$
\begin{aligned}
& g: S \longrightarrow Q_{n-2}=\left\{z \in C P^{n-1} \mid \sum_{\imath} z_{\imath}^{2}=0\right\} \\
& g(w)=\frac{\partial X}{\partial w}=\left(\frac{\partial X_{1}}{\partial w}, \frac{\partial X_{2}}{\partial w}, \cdots, \frac{\partial X_{n}}{\partial w}\right)
\end{aligned}
$$

where $w=u_{1}+i u_{2}$ is a local coordinate of $S$.

By definition a surface $M$ is minimal if

$$
\Delta X_{2}=0 \quad \text { for } \quad i=1,2, \cdots, n
$$

where $\Delta=\frac{\partial^{2}}{\partial u_{1}^{2}}+\frac{\partial^{2}}{\partial u_{2}^{2}}$.

It is known that $g(w)$ is holomorphic iff $M$ is a minimal surface. (see [5]) In this paper we exclude the case where $M$ is a plane.

Let $C P^{n-1}$ have the Fubini-Study metric with 'constant holomorphic curvature 2 ;

$$
d s^{2}=\frac{2 \sum_{j<k}\left|z_{j} d z_{k}-z_{k} d z_{j}\right|^{2}}{\left[\sum_{j=1}^{n}\left|z_{j}\right|^{2}\right]^{2}}
$$

Let $\hat{K}(p)$ denote the Gaussian curvature of $g(S) \subset Q_{n-2} \subset C P^{n-1}$ at a point $p \in S$. It follows immediately that

$$
\hat{K}(p) \leqq 2
$$




\section{Results.}

Let $M$ be a minimal surface in $R^{n}$ with $\hat{K} \equiv c$ (constant). Then Calabi's results tell us that $c$ must be the form $2 / k, k \in N$, and it must satisfy

$$
k \leqq n-1 \text {. }
$$

And furthermore, $g(S)$ must be represented locally

$$
{ }^{t} g(w)=U y_{k}
$$

where $U$ denotes an $n \times n$ unitary matrix, and

$$
y_{k}=\left(1, \sqrt{\left(\begin{array}{c}
k \\
1
\end{array}\right)} w, \sqrt{\left(\begin{array}{c}
k \\
2
\end{array}\right)} w^{2}, \cdots, \sqrt{\left(\begin{array}{l}
k \\
k
\end{array}\right)} w^{k}, 0, \cdots, 0\right) .
$$

From the fact that $g(S) \subset Q_{n-2}, g(w)$ must satisfy

It is equivalent to

$$
g(w) \cdot{ }^{t} g(w)=0
$$

$$
{ }^{t} y_{k}{ }^{t} U U y_{k}=0
$$

Now we set

$$
{ }^{t} U U=A=\left(a_{\imath j}\right) \quad i, j=1, \cdots, n .
$$

Here $A$ is a symmetric unitary matrix. So, $a_{\imath \jmath}=a_{j i}$.

THEOREM A. For every positive integer $m$, there exists a minimal surface with $\hat{K} \equiv 1 / m$ in $2 m+1$ dimensional Euclidean space.

Proof. From the fact

$$
\left(\begin{array}{l}
k \\
0
\end{array}\right)-\left(\begin{array}{c}
k \\
1
\end{array}\right)+\left(\begin{array}{l}
k \\
2
\end{array}\right)-\cdots+(-1)^{j}\left(\begin{array}{l}
k \\
k
\end{array}\right)+\cdots+(-1)^{k}\left(\begin{array}{l}
k \\
k
\end{array}\right)=0,
$$

the matrix

$$
\left(\begin{array}{cccccc}
0 & 0 & 0 & \cdots & 0 & -1 \\
0 & 0 & 0 & & 1 & 0 \\
0 & 0 & & -1 & 0 & 0 \\
& & \cdots & & & \\
0 & 0 & -1 & & 0 & 0 \\
0 & 1 & 0 & & 0 & 0 \\
-1 & 0 & \cdots & 0 & 0 & 0
\end{array}\right)
$$


satisfies the properties of $A$. Because it is a real orthogonal matrix, it is diagonalizable in the sense of real matrices, and its eigenvalues are 1 or -1 . So, the unitary matrix $U$ is easily calculated. q. e. d.

THEOREM B. For every integer $m \geqq 5$, there exists a minimal surface with $\hat{K} \equiv 2 /(2 m-1)$ in $2 m$ dimensional Euclidean space.

Proof. Let

$$
P(j)=\sum_{i=0}^{j}\left(\begin{array}{c}
2 m-1 \\
i
\end{array}\right), \quad Q(j)=\sum_{i=j+1}^{m-2}\left(\begin{array}{c}
2 m-1 \\
i
\end{array}\right) .
$$

There exists $j_{0}$ s.t. $P\left(\jmath_{0}\right) \leqq Q\left(\jmath_{0}\right)$, and $P\left(j_{0}+1\right) \geqq Q\left(j_{0}+1\right)$. Now set

$$
P=P\left(\jmath_{0}\right), \quad Q=Q\left(j_{0}\right), \quad R==\left(\begin{array}{c}
2 m-1 \\
m-1
\end{array}\right) \text {. }
$$

These $P, Q$ and $R$ satisfy the triangle inequality. So, there exist two real numbers $\theta, \varphi$ s.t.

$$
P+Q e^{i \theta}+R e^{\imath \varphi}=0
$$

Let us define the symmetric unitary matrix $A$ as follows;

$$
\begin{aligned}
& a_{s, 2 m+1-s}=\left\{\begin{array}{lll}
1 & \text { for } 1 \leqq s \leqq j_{0}+1 & \text { or } 1 \leqq 2 m+1-s \leqq j_{0}+1 \\
e^{i \theta} & \text { for } j_{0}+2 \leqq s \leqq m-1 & \text { or } j_{0}+2 \leqq 2 m+1-s \leqq m-1 \\
e^{\imath \varphi} & \text { for } s=m, m+1
\end{array}\right. \\
& a_{s, t}=0 \quad \text { for } t \neq 2 m+1-s
\end{aligned}
$$

It is easy to see that the matrix $A$ is decomposed as

$$
A={ }^{t} U U
$$

where $U$ is a unitary matrix. Then, from (3), the equation (2) is satisfied.

q. e. d.

THEOREM C. Let $k=3,5$, or 7 . Then there exists a minimal surface $M$ with $\hat{K} \equiv 2 / k$ in $k+3$ dimensional Euclidean space.

Proof. In this case the matrix $A$ is given as follows;

$$
\begin{aligned}
& \text { for } i+j=k+2, \quad i \neq(k+1) / 2,(k+3) / 2, \quad a_{\imath \jmath}=1 \text {, } \\
& \text { for }(i, j)=((k+1) / 2,(k+3) / 2),((k+3) / 2,(k+1) / 2) \text {, } \\
& a_{\imath \jmath}=\alpha=\left\{\sum_{r=0}^{m-2}\left(\begin{array}{l}
k \\
r
\end{array}\right)\right\} /\left(\begin{array}{c}
k \\
m-1
\end{array}\right)<1 \quad \text { where } m=(k+1) / 2, \\
& \text { for }(i, j)=((k+1) / 2, k+2),((k+3) / 2, k+3),(k+2,(k+1) / 2) \text {, } \\
& (k+3,(k+3) / 2), \quad a_{\imath \jmath}=\sqrt{1-\alpha^{2}},
\end{aligned}
$$




$$
\text { for }(i, j)=(k+2, k+3),(k+3, k+2), \quad a_{\imath \jmath}=-\alpha,
$$

otherwise, $a_{\imath \jmath}=0$.

q. e. d.

Now, we know Calabi's inequality (1) is best possible when $k \neq 1,3,5,7$. And when $k=1,3$, the minimum $n$ is 4,6 respectively. But when $k=5,7$, the minimum $n$ are unknown. In other words, it is unknown whether minimal surfaces with $K=2 / 5(2 / 7)$ exist in $R^{6}, R^{7}$ (in $R^{8}, R^{9}$ respectively), or not.

Remark 1. In theorem A., if $n=3$, then $\hat{K} \equiv 1$ and matrix $A$ must be the form;

And

$$
\left(\begin{array}{rrr}
0 & 0 & -1 \\
0 & 1 & 0 \\
-1 & 0 & 0
\end{array}\right) .
$$

$$
U=\left(\begin{array}{ccc}
1 / 2 & 0 & -1 / 2 \\
i / 2 & 0 & i / 2 \\
0 & 1 & 0
\end{array}\right)
$$

(mod orthogonal transformations in $R^{3}$ ).

From this, we can obtain classical Weierstrass-Enneper's expression formula for classical minimal surfaces.

Remark 2. Also in theorem A., if $n=5$, then $\hat{K} \equiv 1 / 2$ and

$$
U=\left(\begin{array}{ccccc}
1 / \sqrt{2} & 0 & 0 & 0 & -1 / \sqrt{2} \\
i / \sqrt{2} & 0 & 0 & 0 & \imath / \sqrt{2} \\
0 & 1 / \sqrt{2} & 0 & 1 / \sqrt{2} & 0 \\
0 & i / \sqrt{2} & 0 & -i / \sqrt{2} & 0 \\
0 & 0 & i & 0 & 0
\end{array}\right)
$$

(mod orthogonal transformations in $R^{5}$ )

Combining the fact that no minimal surfaces with $\hat{K} \equiv 2 / 3$ exist in $R^{5}$, Masal'tsev's theorem is obtained. (see [4])

\section{REFERENCES}

[1] E. CALABI, Isometric imbeddings of complex manifolds, Ann. of Math., 58 (1953), $1-23$.

[2] H.B. Lawson Jr., Some intrinsic characterizations of minimal surfaces, J. d'Anal. 
Math., 24 (1971), 151-161.

[3] H.B. Lawson Jr., Lectures on Minimal Submanifolds, Vol. 1, Publish or Perish, Inc., (1980).

[4] L. A. Masal'tsev, Minimal Surfaces in $R^{5}$ Whose Gauss Images Have Constant Curvature, Math. Notes of Academy of Science USSR, 35 (1984), 487-490.

[5] R. OsSERman AND D. HOFFMAN, The geometry of the generalized Gauss map, Mem. of Amer. Math. Soc., 236 (1980). 